\title{
The cell-autonomous clock of VIP receptor VPAC2 cells drives circadian behaviour
}

Ryan Hamnett ${ }^{1}$, Johanna E. Chesham ${ }^{1}$, Elizabeth S. Maywood ${ }^{1}$, Michael H. Hastings ${ }^{1}$

1Division of Neurobiology, MRC Laboratory of Molecular Biology, Francis Crick Avenue, Cambridge, Cambridgeshire, UK, CB2 0QH

Corresponding author(s): Ryan Hamnett, rhamnett@stanford.edu; Michael H. Hastings, mha@mrc-Imb.cam.ac.uk

Conflict of Interest: The authors declare no competing financial interests.

\section{Acknowledgements:}

The Authors thank Staff of the MRC LMB Biomedical Facility, Ares, for mouse breeding and handling, Staff of the Light Microscopy Facility for imaging support, and Emma Morris for experimental assistance. This work was funded by the Medical Research Council (MC_U105170643) core funding to MHH.

$\mathrm{RH}$ current affiliation: Department of Neurosurgery, Stanford University, Stanford, CA 94305, USA. 


\section{Abstract}

Circadian ( daily) rhythms pervade mammalian behaviour. They are generated by cell-autonomous, transcriptional/translational feedback loops (TTFL), active in all tissues. This distributed clock network is co-ordinated by the principal circadian pacemaker, the hypothalamic suprachiasmatic nucleus (SCN). Its robust and accurate time-keeping arises from circuit-level interactions that bind its individual cellular clocks into a coherent time-keeper. Cells that express the neuropeptide vasoactive intestinal peptide (VIP) mediate retinal entrainment of the SCN, and in the absence of VIP, or its cognate receptor VPAC2, circadian behaviour is compromised because SCN cells cannot synchronise. The contributions to SCN pacemaking and circadian behaviour of other cell types, not least the VPAC2-expressing target cells of VIP, are, however, not understood. We therefore employed intersectional genetics to manipulate the cell-autonomous TTFL of VPAC2-expressing cells, creating temporally chimaeric mice. We could then determine whether and how VPAC2-expressing cells (a minority $~ 35 \%$ of SCN cells) contribute to SCN timekeeping. Lengthening of the intrinsic TTFL period of VPAC2 cells by deletion of the $C K 1 \varepsilon^{T a u}$ allele concomitantly lengthened the period of circadian behavioural rhythms. It also increased the variability of the circadian period of bioluminescent TTFL rhythms in SCN slices recorded ex vivo. Abrogation of circadian competence in VPAC2 cells by deletion of Bmal1 severely disrupted circadian behavioural rhythms and compromised TTFL time-keeping in the corresponding SCN slices. Thus, VPAC2-expressing cells are a distinct, functionally powerful subset of the SCN circuit, contributing to computation of 
bioRxiv preprint doi: https://doi.org/10.1101/2020.08.02.232462; this version posted Auqust 3.2020. The copvriaht holder for this preprint (which was not certified by peer review) is the author/funder, who has granted bioRxiv a license to display the preprint in perpetuity. It is made available under aCC-BY-NC 4.0 International license.

ensemble period and maintenance of circadian robustness. These findings extend our understanding of SCN circuit topology. 


\section{Introduction}

Mammalian physiology and behaviour are rhythmic and adaptively aligned with the environmental light-dark cycle by the suprachiasmatic nucleus (SCN) of the hypothalamus, which is entrained by direct retinal innervation (Reppert and Weaver, 2002). Within the 20,000 "clock" cells of the SCN, and indeed within most cells across the body, cell-autonomous circadian timekeeping is maintained by a transcriptional/translational feedback loop (TTFL) in which CLOCK:BMAL1 heterodimers activate transcription of Period1/2 and Cryptochrome1/2 genes, the protein products of which (PER1/2 and CRY1/2) feedback to repress their own transcription. This cycle takes approximately 24 $\mathrm{h}$ to complete, although the period of the SCN can be modified considerably by genetic or pharmacological manipulation (Patton et al., 2016), such as the Tau mutation in casein kinase 1 epsilon (CK1 $\left.\varepsilon^{T a u}\right)$, which shortens it to 20 hours in homozygous mice (Meng et al., 2008). More dramatically, mice lacking BMAL1 $\left(B m a / 1^{-/-}\right)$, the only non-redundant TTFL component, are arrhythmic at cellular and behavioural levels (Bunger et al., 2000): effects that are reversible by transgenic rescue (McDearmon et al., 2006).

Beyond the cell-autonomous clock, SCN pacemaking is a product of circuitlevel interactions that bind the circadian cycles of the individual cells. These interactions confer onto the network its essential emergent properties of robust, high-amplitude and synchronised cellular oscillations, with established ensemble phase and period (Hastings et al., 2018). Indeed, the power of intercellular coupling can partially compensate for various genetic losses within 
the TTFL (Liu et al., 2007; Ko et al., 2010). Although GABA is the principal neurotransmitter of the SCN, the strongest evidence for neurochemical mediation of intercellular coupling is not for GABA-ergic signalling but, rather, for a hierarchy of neuropeptides (Maywood et al., 2011). Across the SCN, discrete populations of cells are characterised by their expression of, inter alia, vasoactive intestinal peptide (VIP), arginine vasopressin (AVP), gastrinreleasing peptide (GRP), prokineticin2 (Prok2), and their cognate receptors (Abrahamson and Moore, 2001; Antle and Silver, 2005; Park et al., 2016). These cell-types show a highly stereotypical spatial organization within the SCN, with VIP and GRP cells in the retinorecipient "core", and AVP and Prok2 cells in its surrounding "shell". Mice or SCN slices deficient in intercellular communication mediated by VIP and its receptor VPAC2 (encoded by the Vipr2 gene), display weakened rhythmicity, fewer rhythmic neurons, and damped and desynchronised cellular oscillations (Harmar et al., 2002; Colwell et al., 2003; Aton et al., 2005; Maywood et al., 2006; Ciarleglio et al., 2009). Moreover, VIP cells receive the retinal information that entrains the SCN to solar time (Abrahamson and Moore, 2001; Jones et al., 2018; Mazuski et al., 2018). In turn, VIP acts via the VPAC2-expressing cells of the SCN shell to maintain steady-state circuit-level coherence and to reset ensemble phase in response to retinal input. It achieves this via a cascade of kinase-dependent signalling (including ERK1/2 and its regulator DUSP4) and consequent regulation of a broad transcriptional network (Hamnett et al., 2019). VIP is thereby able to control both cell-autonomous and circuit-level circadian oscillations within the SCN. 
The VIP/VPAC2 axis is, therefore, a central element of SCN circuit topology. Although some functions of VIP cells are established, the functions of their target cells expressing VPAC2, which constitute the next step in the SCN synaptic circuitry, are not. Located in the SCN shell, they may mediate circadian output from the SCN, and/or they may contribute to the circuit-level computations that generate its emergent properties. To investigate this, we employed transgenic mice in which VPAC2 cells express Cre recombinase (Patton et al., 2020). This allowed conditional manipulation of the cellautonomous TTFL of VPAC2-expressing cells, altering their intrinsic period by deletion of $C K 1 \varepsilon^{T a u}$, or their circadian competence by deletion of Bmal1. By monitoring the consequences for behaviour and SCN pacemaking, we reveal that VPAC2-expressing cells are a distinct, functionally powerful subset of the SCN circuit, contributing to computation of ensemble period and maintenance of robustness. These findings extend our understanding of SCN circuit topology. 


\section{Materials \& Methods}

\section{Animals}

All animals were cared for in accordance with the UK Animals (Scientific Procedures) Act of 1986 with local ethical approval (LMB Animal Welfare and Ethical Review Body). VPAC2-Cre mice (Tg(Vipr2cre)KE2Gsat/Mmucd; RRID:MMRRC_034281-UCD) were purchased from GENSAT (Gene Expression in the Nervous System Atlas) project (Rockefeller University, New York City, USA). These mice were subsequently crossed with either $C k 1 \varepsilon^{T a u / T a u}$ (Meng et al., 2008) or Bmal1 ${ }^{f \mid x / f I x}$ mice (generated from Jackson Labs mouse stock no. 007668, RRID:IMSR_JAX:007668). Both the Ck1 $\varepsilon^{\text {Tau/Tau }}$ and

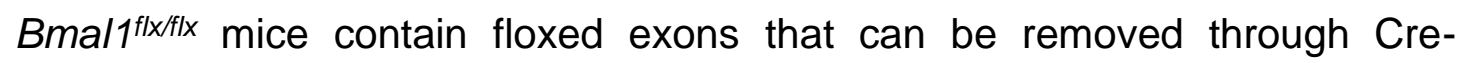
mediated recombination. Due to VPAC2-Cre expression in developing spermatocytes (Usdin et al., 1994; Krempels et al., 1995), which is a known issue with some Cre driver lines (Luo et al., 2020), recombination occurred prior to fertilization, resulting in offspring containing one recombined allele in all cells, alongside the remaining floxed allele to be deleted only in somatic cells expressing VPAC2-Cre. These crosses, therefore, generated VPAC2Cre/Ck1 $\varepsilon^{\text {Tau/- }}$ and VPAC2-Cre/Bmal1 ${ }^{f \mid x /-}$ mice. All mice were also crossed to PER2::LUCIFERASE knock-in mice (gift from Prof Joseph Takahashi (Yoo et al., 2004; University of Texas Southwestern Medical Center, Dallas)) for visualisation of circadian dynamics through bioluminescent recording, and R26R-EYFP mice (Jackson stock 006148, RRID:IMSR_JAX:006148) to report Cre-mediated recombination and determine Bmal1 deletion efficiency (Srinivas et al., 2001). Finally, Drd1a-Cre/Bmal1Flx/- were generated by crossing dopamine 1A-receptor (Drd1a)-Cre (GENSAT, RRID:MMRRC_030779-UCD) 
with Bmal1 ${ }^{f \mid x /}$ mice. This Cre line has previously been shown to have extensive expression in the SCN, covering $63 \%$ of SCN cells and colocalising with $62 \%$ of AVP cells and $81 \%$ of VIP cells (Smyllie et al., 2016). The Drd1a-Cre population has also been shown as being capable of dictating period by crossing with $C k 1 \varepsilon^{T a u} / T a u$ mice. Given its expression profile, it served here as a comparator control for Bmal1 deletion in VPAC2-Cre cells.

\section{Mouse wheel-running behaviour and analysis}

Because the circadian behaviour of adult female mice is modulated by the oestrous cycle, and the oestrous cycle is itself a product of SCN circadian timekeeping, this project used only male mice to avoid potential indirect effects on behaviour arising from SCN-oestrous-behaviour interactions. Male mice were individually housed and kept in a ventilated stainless-steel cabinet with controlled lighting for the duration of behavioural monitoring. Their activity patterns were assessed using running-wheels (Actimetrics Inc.) and passive infrared movement detectors. Mice were typically entrained to a cycle of 12 hours light (L, 200 Ix) and 12 hours dim red light $(D,<10 \mathrm{Lx})(12: 12 \mathrm{LD})$ for at least 7 days to assess entrainment to $24 \mathrm{~h}$ rhythms, before being transferred to constant dim red light (DD) to investigate free-running period. Food and water were provided ad libitum. Wheel revolutions and general movement data were acquired and stored in six-minute bins. Data were analysed using ClockLab v6 (ActiMetrics Inc.; RRID:SCR_014309) with behavioural circadian period in different lighting conditions determined by Chi-squared periodogram. The emergence of the arrhythmic/disordered phenotype in Bmal1-deleted mice was 
determined by eye. The robustness of the rhythms of such mice was quantified using the Relative Amplitude (RA) non-parametric measure in ClockLab v6 on the final 14 days of DD recording.

\section{SCN organotypic slices: bioluminescent recordings and analysis}

SCN organotypic slices and media formulations were prepared as described in Hastings et al. (2005). Briefly, brains were dissected from adult mice and placed into ice-cold dissection medium. SCN tissue was isolated from $300 \mu \mathrm{m}$ slices prepared using a Mcllwain Tissue Chopper (RRID:SCR_015798) and cultured on a Millicell filter membrane (Millipore, RRID:SCR_015799) in $1 \mathrm{ml}$ culture medium. SCN slices acclimatised for $3-6 \mathrm{~h}$ at $37^{\circ} \mathrm{C}, 5 \% \mathrm{CO}_{2}$, and were then transferred to $35 \mathrm{~mm}$ culture dishes containing $1.2 \mathrm{ml}$ recording medium and sealed with glass coverslips, secured with silicon grease, for bioluminescent recordings. Slices were placed under photomultiplier tubes (PMTs; H9319-11 photon-counting head, Hamamatsu) in a light-tight incubator kept at $37^{\circ} \mathrm{C}$ for recording of bioluminescence. These recordings were analysed to calculate circadian period, amplitude and relative amplitude error (RAE; a measure of the rhythm robustness) using the Fast Fourier Transform - Non-Linear Least Squares (FFT-NLLS) function in the BioDare2 software (Zielinski et al., 2014). A 24 h rolling average subtraction was performed on individual traces to account for variable baselines. The first $12 \mathrm{~h}$ of recordings was not included in the analysis to exclude potential artefacts arising from slice preparation. To track the progression of period change in $C k 1 \varepsilon^{T a u}$ experiments, the time between successive PER2::LUCIFERASE peaks was determined. 


\section{Immunohistochemistry and image analysis}

For histological analysis, adult mice were killed rapidly by cervical dislocation and the brains dissected and immediately post-fixed in $10 \mathrm{ml} 4 \%$ paraformaldehyde (Alfa Aesar) in 0.1 M phosphate buffer for 4-5 $\mathrm{h}$ at room temperature, before being cryopreserved in $20 \%$ sucrose (Fisher Chemical) in PBS at $4^{\circ} \mathrm{C}$ overnight. Coronal sections $(40 \mu \mathrm{m})$ were taken using a freezing microtome (Anglia Scientific), incubated for $1 \mathrm{~h}$ at room temperature in $2 \%$ normal serum in PBT (PBS with 1\% bovine serum albumin and $0.3 \%$ Triton $X$ 100 ) then transferred to primary antisera incubation overnight at $4^{\circ} \mathrm{C}$ (see Table 1 for antisera details). Tissue was then incubated with appropriate secondary antibodies (1:500) for $1 \mathrm{~h}$ at room temperature. Sections were mounted onto slides and cover-slipped using Vectashield Hardset mounting medium with DAPI (Vector Labs, RRID:AB_2336788).

\begin{tabular}{|c|c|c|c|c|}
\hline Name & Host & $\begin{array}{l}\text { Supplier and } \\
\text { catalogue number }\end{array}$ & RRID & Concentration \\
\hline Anti-AVP & Rabbit & Penninsula T-4563 & AB_518673 & $1: 1000$ \\
\hline Anti-Bmal1 & Rabbit & $\begin{array}{l}\text { In-house (Reddy et } \\
\text { al., 2006) }\end{array}$ & $\mathrm{N} / \mathrm{A}$ & $1: 500$ \\
\hline Anti-GRP & Rabbit & Immunostar 20073 & $A B \_572221$ & $1: 1000$ \\
\hline Anti-VIP & Rabbit & Immunostar 20077 & AB_572270 & $1: 750$ \\
\hline
\end{tabular}


Fluorescence imaging was conducted using Zeiss 710 and 780 confocal microscopes. Whole SCN sections were imaged using a $20 x$ air objective (NA 0.5 or 0.8 on the 710 and 780 microscopes respectively) while more detailed images (required for cell-counting analysis) were acquired with a $63 x$ oil objective, NA 1.4 before subsequent automated tile-stitching was performed by the Zeiss software (Zen 9 or 10). Counts of cells in SCN sections and determination of fluorescence intensity of SCN neuropeptides were performed in FIJl.

\section{Experimental design and statistical analysis}

Statistical tests and graphical representation of data (mean \pm SEM) were performed using Prism 6 and 7 software (GraphPad). Statistical comparisons were performed using one- or two-way ANOVA with Tukey's multiple comparisons correction, unless otherwise stated. Kruskal-Wallis tests were performed when comparing RA scores between genotypes followed by Dunn's correction for multiple comparisons. Correlation was determined using Pearson's correlation coefficient. Mice and SCN slices were assigned randomly, without regard to genotype, to activity-recording cages and to photomultiplier recording systems, respectively. For both mice in vivo and SCN slices ex vivo, procedures were performed simultaneously on all genotypic groups within an experimental cohort. 


\section{Results}

\section{VPAC2 cells determine the period of the in vivo circadian rhythm of wheel- running behaviour.}

The period-setting potential of VPAC2 cells was examined by generating temporally chimaeric mice using intersectional genetics to delete the Tau allele of the $C k 1 \varepsilon$ gene in VPAC2-expressing cells. Ck1 $1 \varepsilon^{T a u}$ is a semi-dominant point mutation that causes a gain of function for the CK1ع protein that shortens the wild-type behavioural period by $2 \mathrm{~h}$ per copy (heterozygote: $22 \mathrm{~h}$ period;

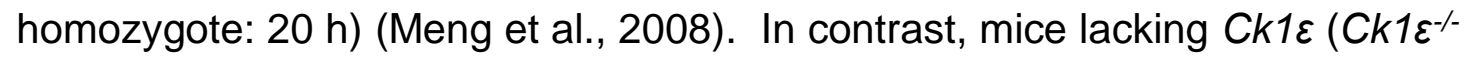
), following Cre-mediated excision of a floxed exon carrying the mutation, have a $24 \mathrm{~h}$ period, whilst $C k 1 \varepsilon^{T a u}$ - exhibit a $22 \mathrm{~h}$ period. Hence, in the VPAC2Cre/Ck1 $1 \varepsilon^{T a u /-m o u s e, ~ V P A C 2-C r e ~ c e l l s ~ w o u l d ~ h a v e ~ a ~ p e r i o d ~ o f ~} 24 \mathrm{~h}$ (being $C k 1 \varepsilon^{-}$ I-), whereas the rest of the SCN and other tissues would retain a period of $22 \mathrm{~h}$ (Fig. 1A). If the cell-autonomous TTFL of VPAC2 cells directs circadian behaviour, such lengthening of their intrinsic period should correspondingly lengthen the period of wheel-running rhythms of the mouse.

The wheel-running behaviour of mice of all genotypes $\left(C k 1 \varepsilon^{W T /-}\right.$ and $C k 1 \varepsilon^{T a u /-}$, without and with VPAC2-Cre) entrained stably to a 12:12 light:dim red light (LD) cycle (Fig. 1B, $C$; two-way ANOVA, $F(1,18)=3.706, p=0.07($ Cre $), F(1,18)=0.000$, $p>0.999(\mathrm{Tau}), F_{(1,18)}=0.000, p>0.999$ (interaction)). On transfer to continuous dim red light (DD), $C k 1 \varepsilon^{W T /-}$ mice free-ran with a wild-type equivalent period $(\sim 24 \mathrm{~h})$, whereas that of $C k 1 \varepsilon^{T a u /-}$ mice was significantly shorter $(\sim 22 \mathrm{~h}$, Fig. $1 B, D$; two-way ANOVA, $F(1,39)=30.46, p<0.0001$ (interaction), Tukey posthoc $p<0.0001)$. The presence of VPAC2-Cre had no effect in $C k 1 \varepsilon^{W T-}$ mice 
A

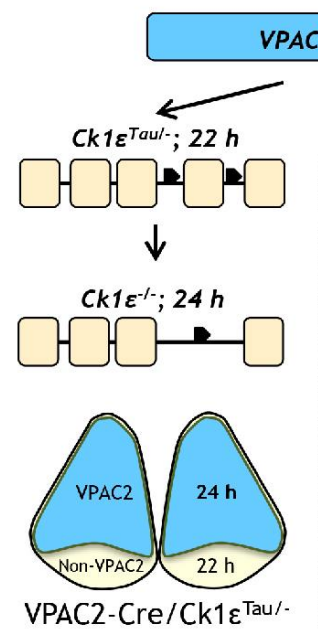

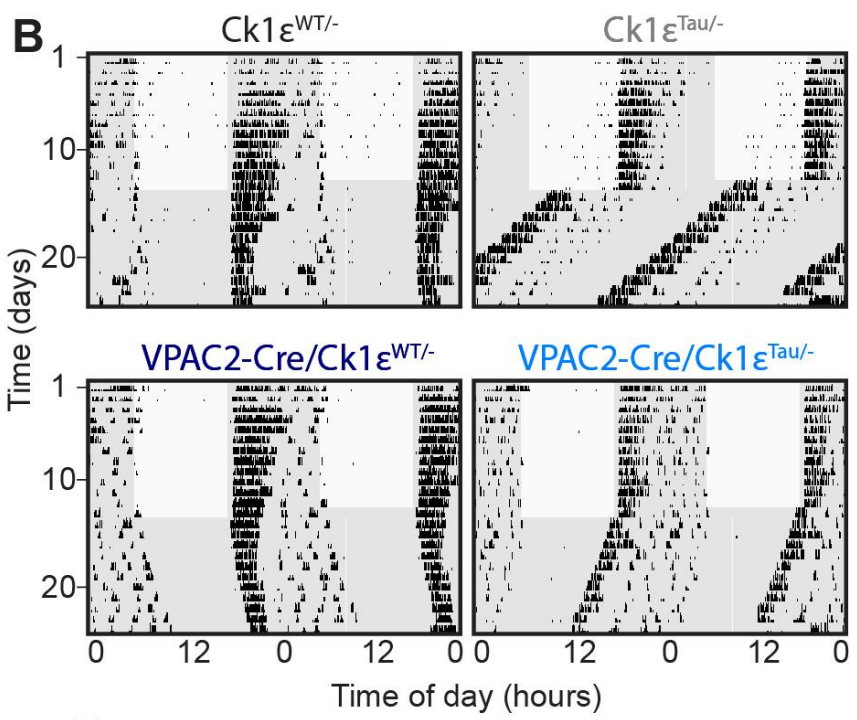

E
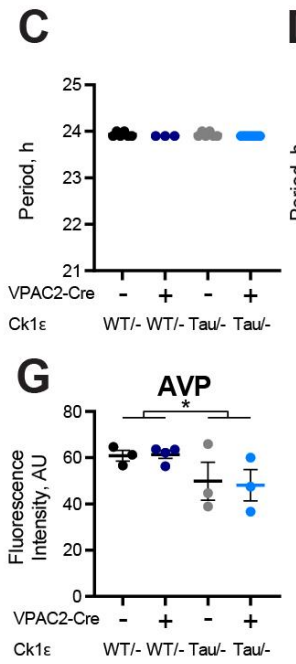

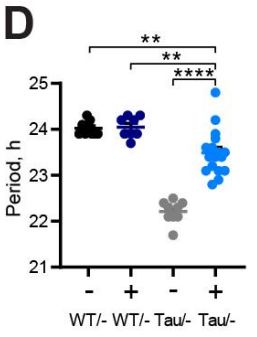

VIP

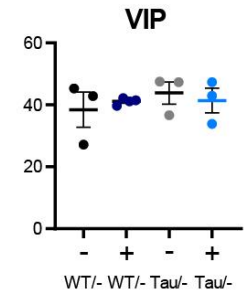

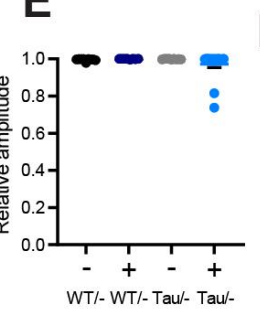

GRP

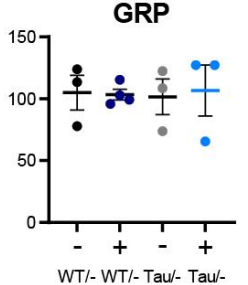

$\mathbf{F}_{\mathrm{Ck} 1 \varepsilon^{\mathrm{WT} /-}}$ VPAC2-Cre
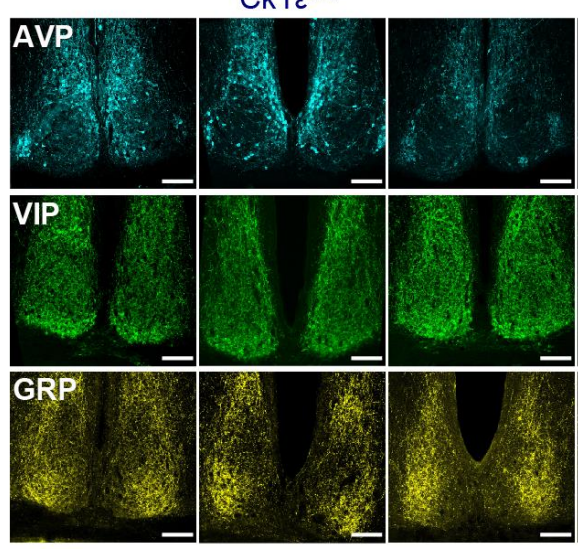

Figure 1. Intersectional genetics reveals that VPAC2-expressing cells determine the circadian period of mouse wheel-running behaviour.

$\boldsymbol{A}$, Schematic view of the intersectional approach, whereby mice carrying Cre recombinase as a transgene controlled by the VPAC2 promoter were crossed with either $C k 1 \varepsilon^{\text {Taul- }}$ or Bmal/ $1^{\text {flx/- }}$ mice to create a chimaeric SCN. The result is for VPAC2 cells to lack either the Tau allele or Bmal1, reverting them to a $24 \mathrm{~h}$ period as $C k 1 \varepsilon^{-/-}$or rendering them arrhythmic as Bmal1 ${ }^{-1}$, respectively. $\boldsymbol{B}$, Representative double-plotted actograms of wheel-running activity of VPAC2Cre/Ck1 $\varepsilon^{\text {Tau/ }}$ mice and their respective controls exposed to a 12:12 light:dim red (LD) cycle followed by continuous dim red light (DD) conditions. Grey shading represents dim red. $\boldsymbol{C}, \boldsymbol{D}$ Periods (mean \pm SEM) observed in 12:12 LD $\left(C ; \mathrm{n}=6\left(C k 1 \varepsilon^{W T /}\right), 3\left(\mathrm{VPAC2}-\mathrm{Cre} / \mathrm{Ck} 1 \varepsilon^{W T /}\right), 6\right.$ $\left(C k 1 \varepsilon^{T a u /}\right)$ and 7 (VPAC2-Cre/Ck1 $\left.\left.\varepsilon^{\text {Tau/ }}\right)\right)$ and DD $(\boldsymbol{D} ; \mathrm{n}=9,8,9,17)$ of VPAC2-Cre/Ck1 $\varepsilon^{\text {Tau/- }}$ mice and their respective controls. $\boldsymbol{E}$, Relative amplitude scores (mean \pm SEM) from 10 days of wheel running activity under DD ( $\mathrm{n}$ as in $\boldsymbol{D}$ ). Kruskal-Wallis test. $\boldsymbol{F}$, Representative images of immunohistochemical staining of SCN of VPAC2-Cre/Ck1 $1 \varepsilon^{\text {Tau/- }}$ mice and their respective controls for AVP-ir (top), VIP-ir (middle) and GRP-ir (bottom). Scale bar represents $100 \mu \mathrm{m}$. $G$, Fluorescence intensities (mean \pm SEM) for immunohistochemical staining of AVP-ir (left), VIP-ir (middle) and GRP-ir (right) in SCN sections of VPAC2-Cre/Ck1 $\varepsilon^{\text {Tau/- }}$ mice with their respective controls ( $n=3$ per genotype). All tests were two-way ANOVAs (main effects of Cre and Tau allele) with Tukey's multiple comparisons test, ${ }^{*} p<0.05,{ }^{* *} p<0.01,{ }^{* * * *} p<0.0001$. 
lacking the floxed allele, whereas its presence in the $C k 1 \varepsilon^{T a u /-}$ mice caused significant lengthening of circadian period (Tukey post-hoc Ck1 $\varepsilon^{\text {Tau/ }}(22.21$ $\pm 0.08 \mathrm{~h})$ vs. VPAC2-Cre/Ck1 $1 \varepsilon^{\text {Tau/- }}(23.49 \pm 0.12$ h) $p<0.0001)$. This VPAC2Cre/Ck1 $\varepsilon^{\text {Tau/- } g r o u p ~ m e a n ~ w a s ~ s l i g h t l y, ~ a l b e i t ~ s i g n i f i c a n t l y, ~ b e l o w ~ t h e ~} 24 \mathrm{~h}$ period of $C k 1 \varepsilon^{W T /-}$ controls, with or without VPAC2-Cre (Tukey post-hoc Ck1 $1 \varepsilon^{W T /-}$ vs. VPAC2-Cre/Ck1 $\left.\varepsilon^{\text {Tau/- }} p=0.004\right)$. Nevertheless, at a behavioural level, the cellautonomous clock of VPAC2 cells can exert a clear influence on circadian period, lengthening it in line with their intrinsic period. Furthermore, the coherence and amplitude of the behavioural rhythm were not affected by temporal chimaerism (Fig. 1E; Kruskal-Wallis test, $\mathrm{H}(3)=3.661, p=0.30$ ), confirming that circuit-level mechanisms are able to sustain coherent circadian output in the face of widely divergent cell-autonomous periods in the SCN (Smyllie et al., 2016; Brancaccio et al., 2017).

To test further the effect of chimaerism on SCN integrity, sections of adult mouse brain were processed for immunohistochemical (IHC) staining of the neuropeptides VIP, AVP and GRP. The morphology of the SCN was comparable across all genotypes and there were no significant differences in the expression of VIP (two-way ANOVA, $F_{(1,9)}=0.001, p=0.97(\mathrm{Cre}), F_{(1,9)}=$ $0.628, p=0.45(\mathrm{Tau}), F_{(1,9)}=0.518, p=0.49$ (interaction)) or GRP (Fig. 1F,G; two-way ANOVA, $F_{(1,9)}=0.016, p=0.90$ (Cre), $F_{(1,9)}=0.000, p=0.99$ (Tau), $F_{(1,9)}=0.062, p=0.81$ (interaction)). Compared to $C k 1 \varepsilon^{W T /-}$ controls, however, the $C k 1 \varepsilon^{T a u /-}$ mice showed a small ( 20\%) but significant reduction of AVP expression (Fig. 1F, G; two-way ANOVA, $F_{(1,9)}=0.013, p=0.91(\mathrm{Cre}), F_{(1,9)}=$ 5.751, $p=0.04$ (Tau), $F(1,9)=0.053, p=0.82$ (interaction)). This was evident in SCN from mice both without and with the VPAC2-Cre and so could not be a 
direct cause of the period-lengthening observed in the latter. Temporal chimaerism did not, therefore, affect the structural integrity of the SCN, nor the quality of behavioural circadian output, and revealed the pace-setting ability of SCN VPAC2 cells.

\section{Period determination by VPAC2 cells is attenuated in the SCN ex vivo}

Following the recording of wheel-running behaviour, mice were killed under dim red light and their SCN PER2-driven bioluminescence rhythms were recorded to determine the impact of temporal chimaerism on the intrinsic SCN TTFL. SCN of all four genotypes exhibited robust and coherent bioluminescent rhythms (Fig. 2A). As with behavioural periods, the rhythms were shortened by approximately $2 \mathrm{~h}$ by the presence of a single copy of the Tau allele in the SCN of mice lacking VPAC2-Cre (Fig. $2 A, B$; two-way ANOVA, $F(1,21)=39.01, p<$ $0.0001(\mathrm{Tau}))$. In contrast to the lengthening of period observed in vivo, however, there was no systematic or significant effect of VPAC2-Cre on the mean circadian period of $C k 1 \varepsilon^{\text {Tau/- }}$ SCN slices (two-way ANOVA, $F_{(1,21)}=0.408$, $p=0.53$ (interaction)), and the mean circadian period was not statistically

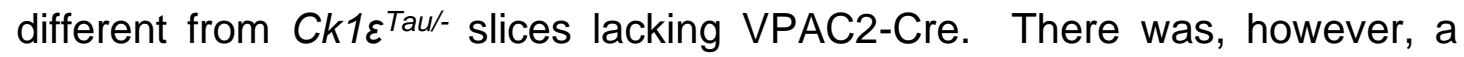
marked variability within the VPAC2-Cre/Ck1 $1 \varepsilon^{T a u}$ - group, with a range of $\sim 6.3$ hours, a clear departure from the behavioural data (Fig. 2B,C). Furthermore, in the control groups there was a direct correspondence between the circadian periods measured in vivo as behaviour and ex vivo as SCN bioluminescence within individual mice, with slices typically having a slightly longer period (Fig. 2C). This was not the case for the VPAC2-Cre/Ck1 $1 \varepsilon^{\text {Tau/- }}$ group, in which the ex 
A

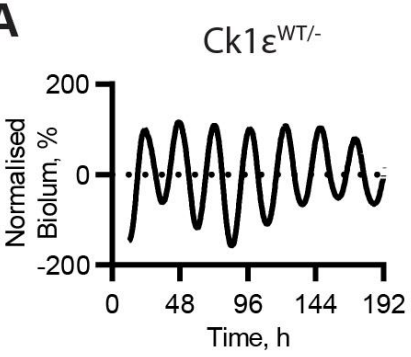

B

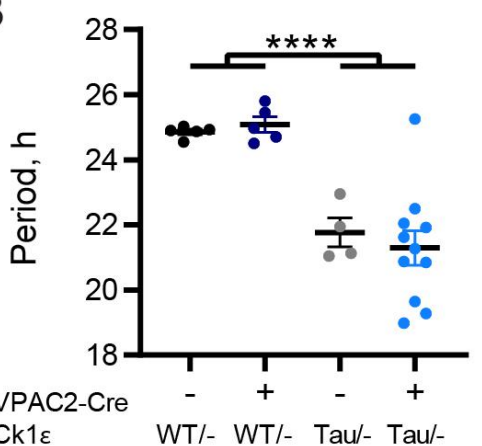

VPAC2-Cre/Ck1 $1 \varepsilon^{\mathrm{WT} /-}$

Ck1 $\varepsilon^{\text {Tau/- }}$

VPAC2-Cre/Ck1 $\varepsilon^{\text {Tau/- }}$
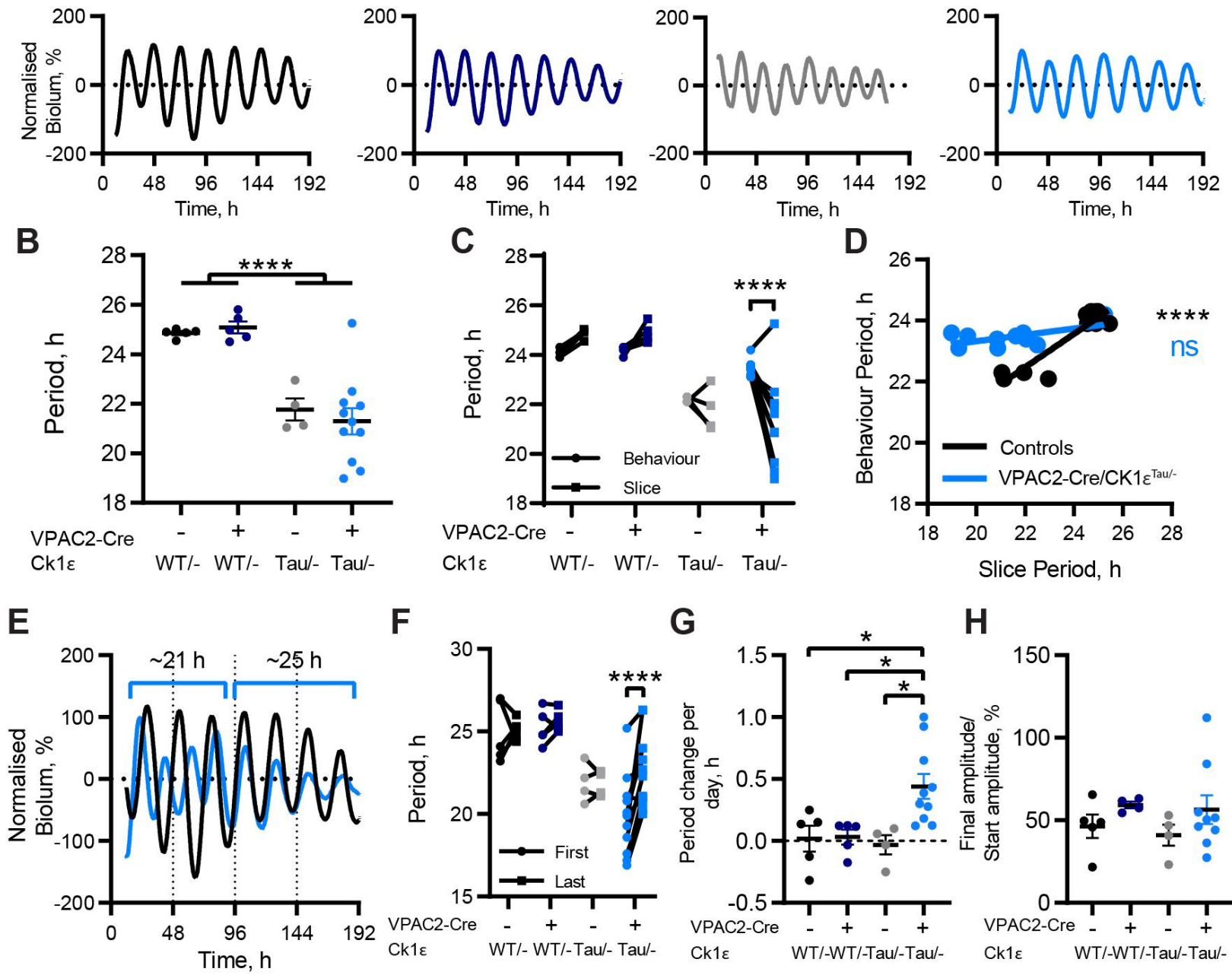

$\mathbf{F}$
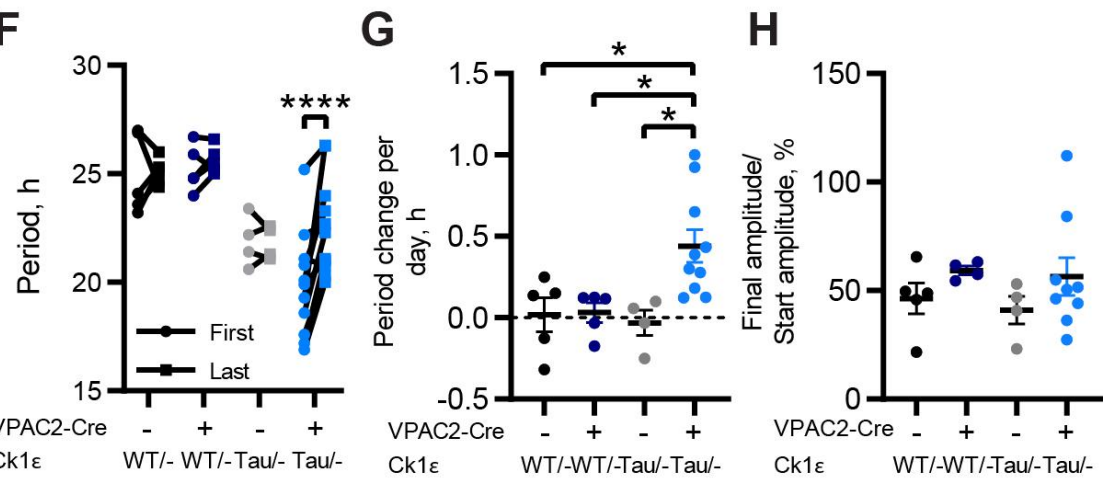

Figure 2. Circadian periods of bioluminescent rhythms of SCN slices from VPAC2-Cre$\mathrm{Ck} 1 \varepsilon^{\mathrm{Tau} /}$ mice do not reflect behavioural period.

A, Representative baseline corrected PER2::LUCIFERASE bioluminescence rhythms from Ck1 $\varepsilon^{W T /}$, VPAC2-Cre/Ck1 $\varepsilon^{W T /}, C k 1 \varepsilon^{\text {Tau/ }}$ and VPAC2-Cre/Ck1 $1 \varepsilon^{\text {Tau/ }}$ SCN dissected following wheel-running recordings. $B$, Periods (mean $\pm S E M$ ) of the first 4 bioluminescent cycles of adult SCN slices as in $\boldsymbol{A}$. $\boldsymbol{C}$, Comparison of circadian periods of free-running behaviour and slice bioluminescence rhythms (measured from the first 4 cycles) from individual mice. $\boldsymbol{D}$, Scatter plot of slice periods (measured from the first 4 cycles) vs. behavioural periods. VPAC2$\mathrm{Cre} / C k 1 \varepsilon^{T a u /-m i c e}$ show no significant correlation ( $p=0.07$, Pearson's correlation) but grouped control slices do $\left({ }^{* * * *} p<0.0001\right.$, Pearson's correlation). Lines represent linear regression: VPAC2-Cre $/ C k 1 \varepsilon^{\text {Tau/- }} \mathrm{r}^{2}=0.36, \mathrm{Y}=0.1037^{\star} \mathrm{X}+21.25$; Controls $\mathrm{r}^{2}=0.85, \mathrm{Y}=0.5458^{*} \mathrm{X}+10.47$. $\boldsymbol{E}$, Representative baseline corrected PER2::LUCIFERASE bioluminescence rhythms from $C k 1 \varepsilon^{W T /}$ and VPAC2-Cre/Ck1 $\varepsilon^{T a u /-S C N}$ slices displaying no phase alignment initially, followed by period lengthening in the VPAC2-Cre/Ck1 $\varepsilon^{T a u /-}$ slice and resultant phase alignment. $\boldsymbol{F}$, Comparison of periods between the first two bioluminescent cycles ('First') and final two cycles ('Last') within each SCN slice. G, Period change per day (mean \pm SEM) in SCN slices. $\boldsymbol{H}$, Bioluminescence amplitude (mean \pm SEM) in the last 3 cycles as a percentage of the amplitude in the first 3 cycles. For $\boldsymbol{B}$ and $\boldsymbol{F}-\boldsymbol{H}, \mathrm{n}=5\left(\mathrm{Ck} 1 \varepsilon^{W T /}\right), 5$ (VPAC2-Cre/Ck1 $\left.1 \varepsilon^{W T-}\right), 4\left(C k^{1 \varepsilon^{T a u} /}\right)$ and 11 (VPAC2-Cre/Ck1 $\left.\varepsilon^{T a u /}\right)$. For $\boldsymbol{C}$ and $\boldsymbol{D}, \mathrm{n}=5\left(C k_{1} \varepsilon^{W T /}\right), 4$ (VPAC2-Cre/Ck1 $\left.\varepsilon^{W T /}\right), 4\left(C k^{1} \varepsilon^{\text {Tau/- }}\right.$ ) and 10 (VPAC2-Cre/Ck1 $1 \varepsilon^{T a u /-)}$. All tests were two-way ANOVAs with Tukey's multiple comparisons test, ${ }^{*} p<0.05,{ }^{* * * *} p<0.0001$. 
vivo SCN periods were not only widely divergent, but were also not consistent with the in vivo period of the corresponding mouse, typically being shorter (Fig. $2 C$; two-way ANOVA, $F_{(3,19)}=8.975, p=0.0007$ (interaction); Sidak's post-hoc

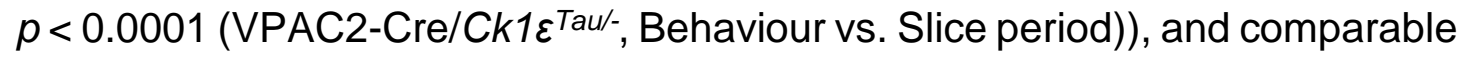
to or even faster than those of $C k 1 \varepsilon^{\text {Tau/- }}$ SCN lacking recombinase. Consequently, when in vivo and ex vivo periods were plotted together, the control groups exhibited a highly significant within-animal correlation but the

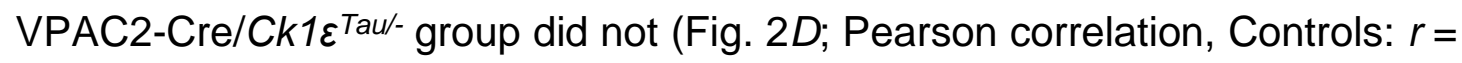
0.923, $p<0.0001$, VPAC2-Cre/Ck1 $\left.\varepsilon^{\text {Tau/-: }} r=0.598, p=0.07\right)$.

The period-lengthening by deletion of $C k 1 \varepsilon^{T a u}$ in VPAC2 cells was therefore consistently effective in vivo but had variable penetrance in the corresponding SCN slices ex vivo. This variability was also evident in the stability of the individual SCN rhythms, whereby the period of VPAC2-Cre/Ck1 $1 \varepsilon^{\text {Tau/- }}$ slices tended to increase over time in culture (Fig. 2E-G; two-way ANOVA, $F_{(3,21)}=$ 4.478, $p=0.014$ (interaction), Tukey post-hoc $p<0.0001$ ), suggesting that the long-period VPAC2 cells were re-exerting influence over ensemble period. While there was considerable variability in the peak-to-peak period within the group (Fig. 2C,F), the average period increase was almost 30 minutes per day for VPAC2-Cre/Ck1 $1 \varepsilon^{\text {Tau/- }}$ slices, whereas control slices did not show significant period-lengthening during ex vivo culture (Fig. 2G; two-way ANOVA, $F_{(1,20)}=$ 4.445, $p=0.048$ (interaction), Tukey post-hoc $p=0.030$ (vs. Ck1 $1 \varepsilon^{W T /}$ ), $p=$ 0.023 (vs. Ck1 $\varepsilon^{\text {Tau/-) }}$, $p=0.035$ (vs. VPAC2-Cre)). Importantly, there was no significant difference in the progressive fall in amplitude observed over time in all genotypes (Fig. $2 H$; two-way ANOVA, $F(1,18)=2.555, p=0.13(\mathrm{Cre}), F_{(1,18)}=$ $0.216, p=0.65$ (Tau), $F(1,18)=0.020, p=0.89$ (interaction)), suggesting that the 
period lengthening was not the result of cells within the slice desynchronising. Overall, we conclude that cell-autonomous properties of VPAC2 cells contribute strongly to computation of ensemble period in vivo, and also, but to a lesser extent, ex vivo.

\section{The cell-autonomous clock of VPAC2 cells is essential for the circadian coordination of rest/activity rhythms.}

To test whether circadian competence in VPAC2 cells is essential for the generation of behavioural rhythms and/or molecular pacemaking in the SCN, we examined the impact of cell-type-specific deletion of BMAL1 by Cremediated removal of a critical exon within a floxed allele of the Bmal1 gene (Fig. 1A). Homozygous loss of Bmal1 results in a severely disrupted or arrhythmic TTFL, although a single floxed copy of Bmal1 is fully functional and sufficient for normal pacemaking (Bunger et al., 2000; Gibbs et al., 2012). All experiments were conducted using Bmal/flx/- mice because the efficiency of Bmal1 deletion in the SCN is enhanced when the floxed Bmal1 allele is paired against a null allele (Husse et al., 2011). The efficiency of deletion in VPAC2expressing cells (constituting $\sim 35 \%$ of SCN cells) was determined by IHC for BMAL1-expression and the expression of a genomic EYFP reporter (RosaLSL-EYFP) to identify Cre-expressing cells (Fig. 3A). VPAC2-Cre-mediated excision reduced the number of BMAL1-expressing cells across the SCN by $\sim 20 \%$ when compared to wild-type SCN (Fig. 3B; one-way ANOVA, $F_{(4,22)}=$ 98.83, $p<0.0001$, Tukey's post-hoc $p<0.0001$ ), which suggests appreciable but incomplete deletion from all VPAC2-expressing cells. Indeed, within the 
A
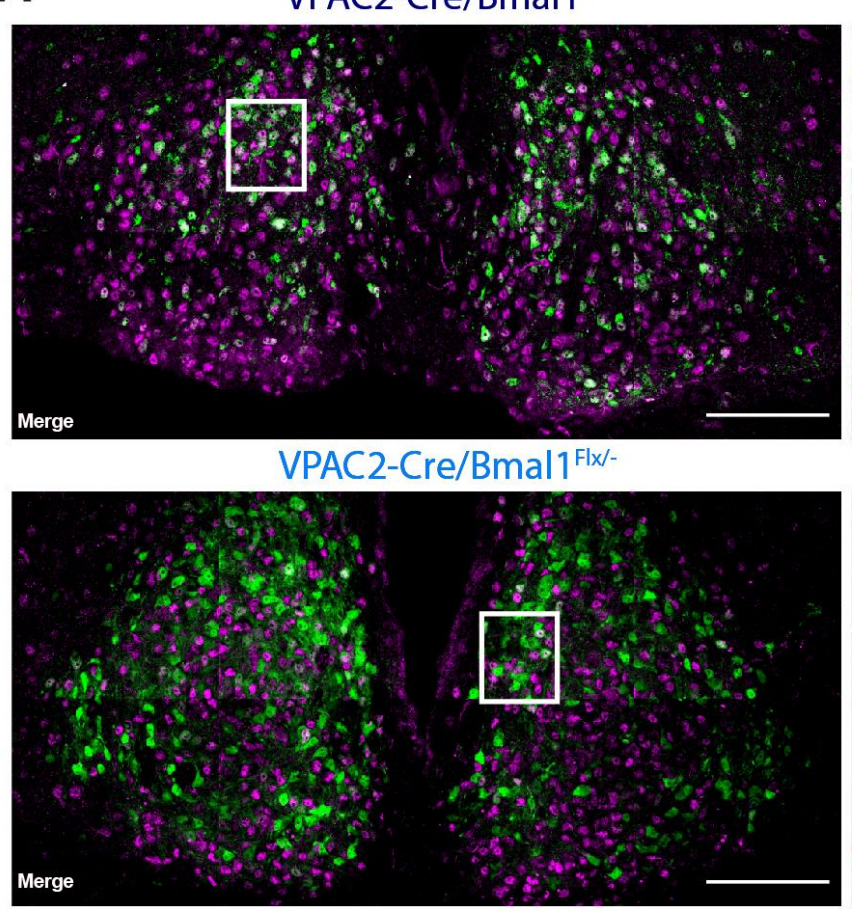

D

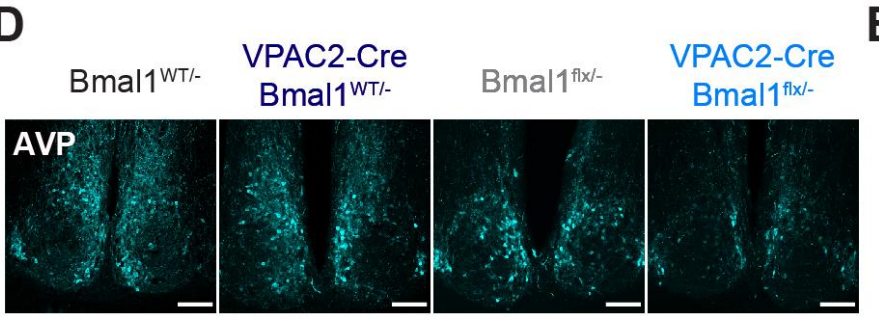

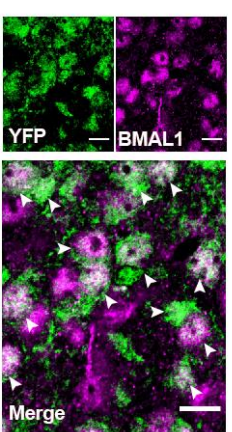

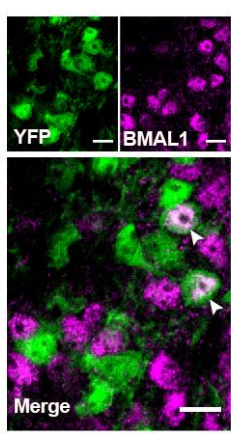

E

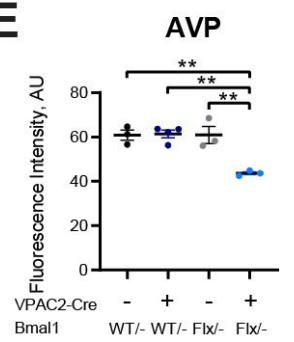

C

\section{B}

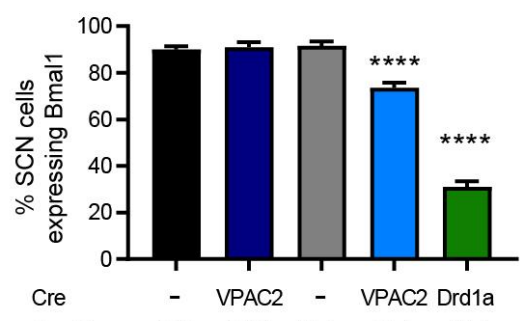

Bmal1

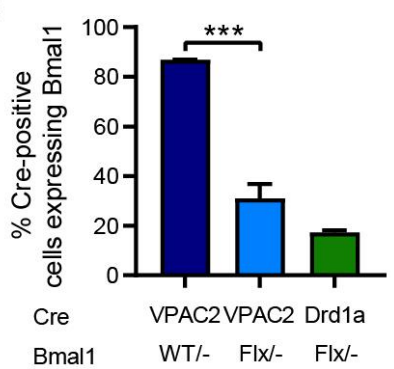

VIP

GRP

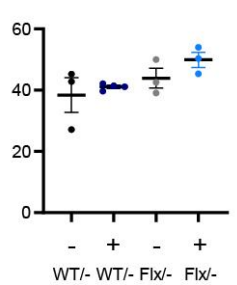

Figure 3. Targeted deletion of BMAL1 from VPAC2-Cre-expressing SCN cells.

$\boldsymbol{A}$, Representative $63 x$ tiled confocal micrographs of Cre recombinase activity, as reported by a genetically encoded EYFP reporter (green), and BMAL1 immunohistochemistry (magenta) in VPAC2-Cre/Bmal1 $1^{W T /}$ and VPAC2-Cre/Bmal/ $1^{f / x /-} \mathrm{SCN}$ sections. Locations of magnified images are indicated by white rectangles. White arrowheads indicate co-localisation between EYFP and BMAL1-ir. Scale bars represent $100 \mu \mathrm{m}$ in stitched images, $10 \mu \mathrm{m}$ in magnified images. $\boldsymbol{B}$, Percentage of SCN cells (marked by DAPI; mean \pm SEM) expressing BMAL1-ir across genotypes, including Drd1a-Cre/Bmal/ $1^{f l x /}$. C , Percentage of Cre-positive cells (marked by EYFP; mean \pm SEM) expressing BMAL1-ir across genotypes. ( $\boldsymbol{B}$, $\boldsymbol{C}$; One-way ANOVA with Tukey's multiple comparisons test). $\boldsymbol{D}$, Representative images of AVP-ir in SCN

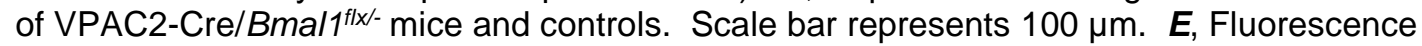
intensities (mean \pm SEM) for immunohistochemical staining of AVP (left), VIP (middle) and GRP (right) in SCN sections of VPAC2-Cre/Bmal/ $1^{f(x)}$ mice and controls. For $\boldsymbol{B}$ and $\boldsymbol{C}, \mathrm{n}=7$

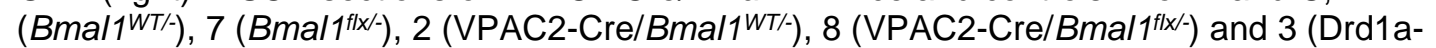
Cre/Bmal/ $\left.f^{f(x /}-\right)$. For $E, \mathrm{n}=3$ per group. Two-way ANOVA with Tukey's post-hoc test. ${ }^{* *} p<$ $0.01,{ }^{* * *} p<0.001,{ }^{* * * *} p<0.0001$. 
VPAC2-Cre expressing cell population (labelled by EYFP), 30\% of cells had detectable BMAL1-ir. This nevertheless emphasised the efficient and specific targetting of BMAL1 in $\sim 70 \%$ of VPAC2 cells (Fig. $3 C$ ). Importantly, the loss of BMAL1 from VPAC2-expressing cells did not affect overall SCN morphology or expression of VIP or GRP (Fig. 3E). There was, however, a small ( $30 \%)$ but significant decline in AVP-ir (Fig. 3D, E; two-way ANOVA, $F_{(1,9)}=14.34, p=$ 0.004 (interaction), Sidak's post-hoc $p=0.004$ (vs. Bmal1F/x/),$p=0.002$ (vs. VPAC2Cre/Bmal1WT/-)), consistent with some cells co-expressing AVP and VPAC2 (Patton et al., 2020; Wen et al., 2020), and the Avp gene being a circadian clock-controlled target of the BMAL1-dependent TTFL (Jin et al., 1999; Mieda et al., 2015).

Mice of all genotypes: Bmal1WT/- and Bmal1F/x/- both without and with VPAC2Cre, entrained stably under a 12:12 LD cycle with a period of $24 \mathrm{~h}$ and there were no obvious differences in activity patterns between them (Fig. 4A; twoway ANOVA, $F_{(1,29)}=0.459, p=0.5(\mathrm{Cre}), F_{(1,29)}=0.672, p=0.42$ (Bmal1), $F(1,29)=2.81, p=0.10$ (interaction)). Mice were then transferred to DD for up to 7 weeks to allow the emergence of any Bmal1\%-dependent phenotypes. Control mice (Bmal1 ${ }^{W T /}$ - with or without VPAC2-Cre, and Bmal1F/x/- without Cre) free-ran with clear circadian patterns and endogenous periods slightly longer than $24 \mathrm{~h}$ (Fig. 4B,C) and with well-defined amplitude (Fig. 4E). The VPAC2Cre/Bmal1 $1^{f \mid x /-}$ group, however, displayed highly variable phenotypes both between and within individual animals under DD (Fig. 4C). Thirteen of 18 mice showed a strong phenotype: arrhythmicity occurred in 5 mice, while fragmented or "split" behaviour was the most common result, seen in 8 mice, with several 
A

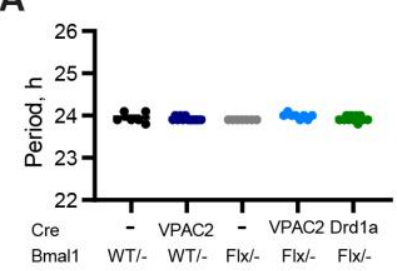

B

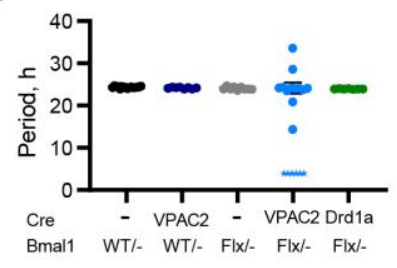

C
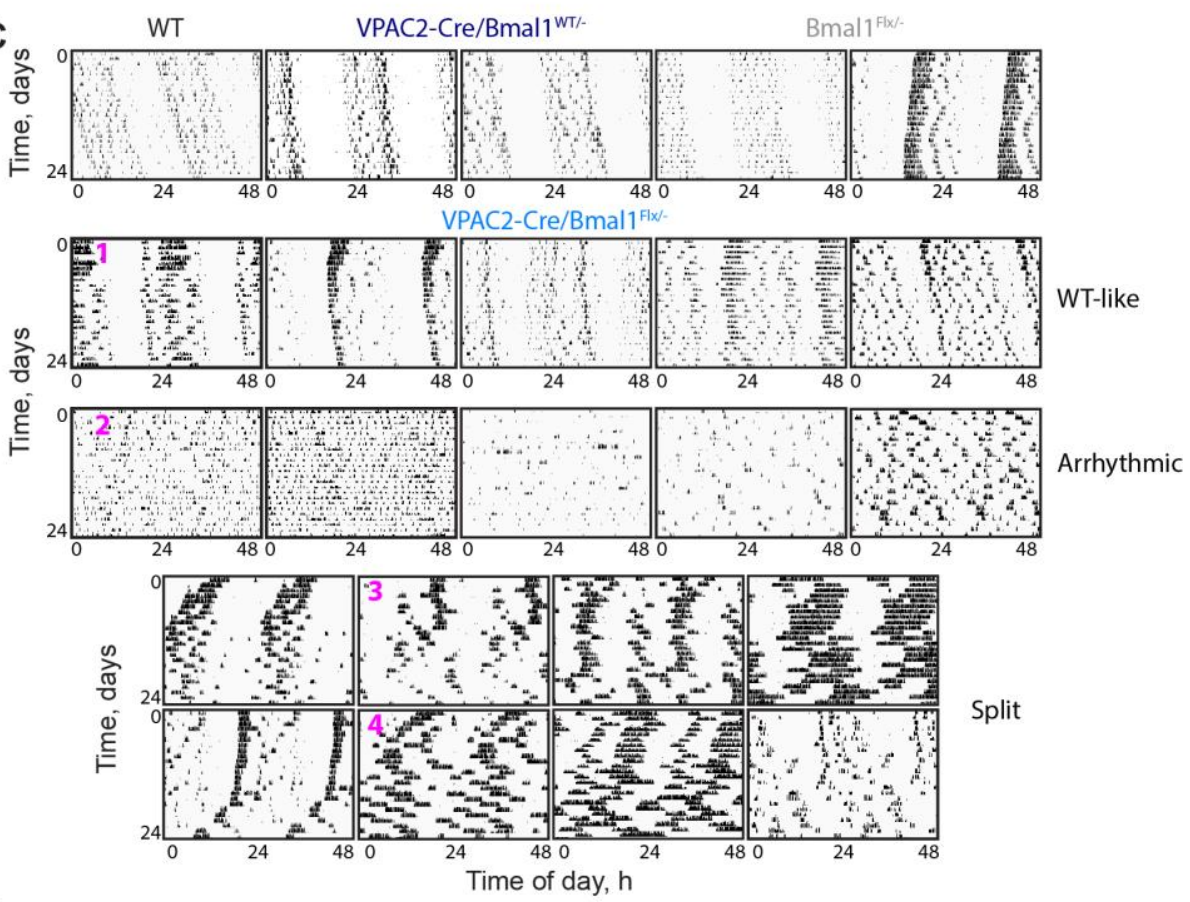

D
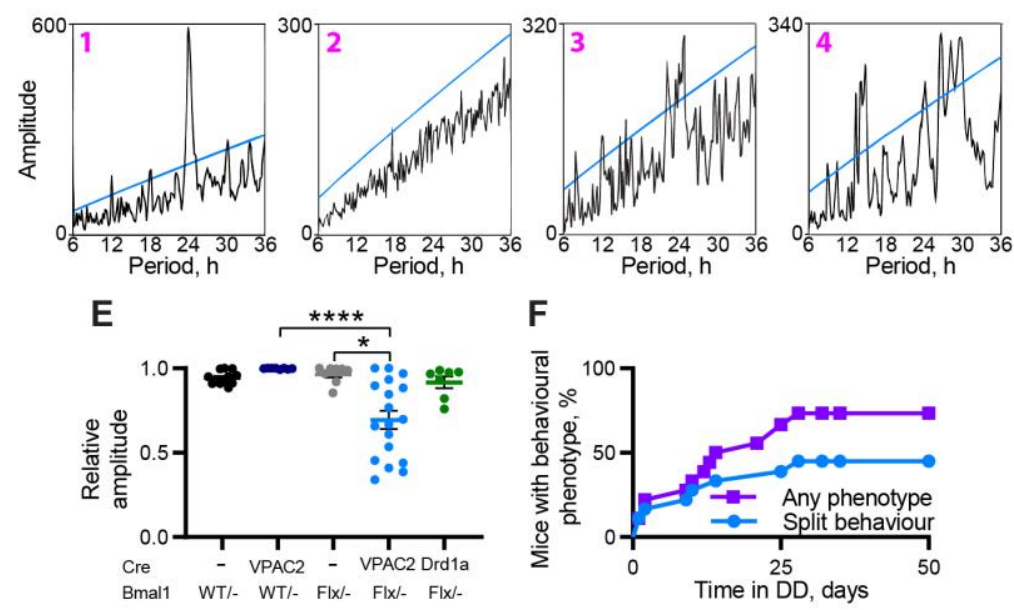

G

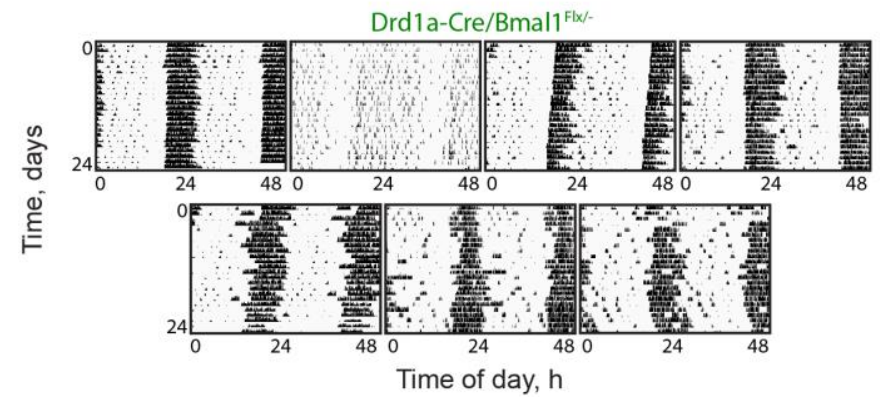

Figure 4. Deletion of BMAL1 from VPAC2-Cre-expressing cells compromises circadian behaviour. (legend continued on next page) 
$\boldsymbol{A}, \boldsymbol{B}$, Circadian periods (mean \pm SEM) of VPAC2-Cre/Bmal ${ }^{f \mid x /}$ mice and controls under $(\boldsymbol{A})$ $12: 12 \mathrm{LD}$ and $(\boldsymbol{B})$ DD. For 6 VPAC2-Cre/Bmal $1^{f / x /}$ mice an explicit single period could not be determined due to their disturbed behaviour and so these are added as star symbols as a nominal 3 hours, and were excluded from statistical analysis. Two-way ANOVA (excluding

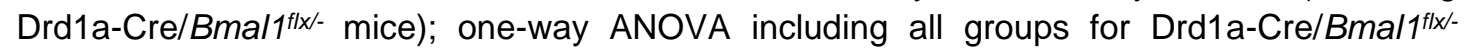
comparison. $\boldsymbol{C}$, Representative double-plotted actograms of final 24 days of wheel-running

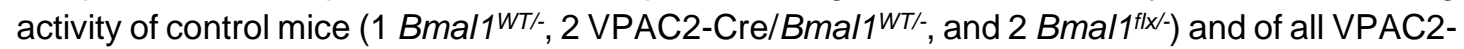
$\mathrm{Cre} / B m a / 1^{f \mid x /}$ mice. VPAC2-Cre/Bmal ${ }^{1 \mid x /}$ - actograms are divided into three groupings (WT-like, Arrhythmic, and Split) and then ranked in descending order of relative amplitude score under DD (final 14 days of recording). $D$, Chi-square periodograms for 4 VPAC2-Cre/Bmal $1^{f(x /}$ - as indicated in $\boldsymbol{C}$. (1) has a single significant peak at $24 \mathrm{~h}$, (2) has no significant period, and (3) and (4) display multiple significant periods. $\boldsymbol{E}$, Relative amplitude scores (mean $\pm S E M$ ) from the last 14 days of wheel running activity under DD. Kruskal-Wallis test with Dunn's multiple

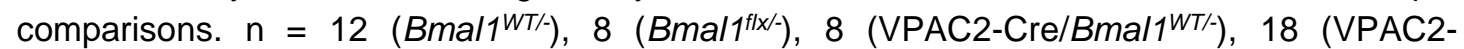

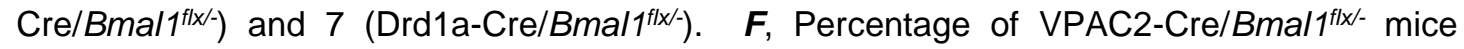
displaying behavioural phenotypes (purple, squares) or specifically split behaviour (blue, circles) over time. $G$, Double-plotted actograms of wheel-running activity of all Drd1aCre/Bmal/1|1/x/- mice. ${ }^{*} p<0.05,{ }^{* * *} p<0.0001$. 
animals displaying multiple significant periods (Fig. 4B-D). Furthermore, in the majority of mice, the amplitude of the activity rhythm was reduced, leading to a significant difference with control groups (Fig. 4E; Kruskal-Wallis test, $H(3)=$ 21.19, $p<0.0001$, Dunn's post hoc $p<0.0001$ (vs. VPAC2-Cre/Bmal1wT/-), $p=$ 0.032 (vs. Bmal1F/x/-)). Strikingly, BMAL1-dependent phenotypes (arrhythmicity, multiple periodicities, loss of amplitude) did not appear at a consistent point following transfer to DD. Rather, they accumulated progressively (Fig. 4F), at a range of times throughout the experiment: some mice immediately showed split or arrhythmic behaviour, while others took several weeks and displayed phenotypes that looked wild-type until that point. We conclude that the cell-autonomous rhythmicity of VPAC2-expressing cells is essential for normal circadian control of behaviour, though considerable plasticity exists within and between individual mice that affects the precise timing and presentation of the VPAC2-Cre/Bmal/flx/- phenotype.

To determine whether the behavioural effects of the local loss of BMAL1 in VPAC2 cells were specific to that cell type, an additional group of mice was included in which BMAL1 was deleted from cells expressing Cre driven by the Drd1a promoter. In these animals, BMAL1 was deleted in $\sim 80 \%$ of Drd1aspecific cells compared to $\sim 70 \%$ of VPAC2-expressing cells in VPAC2Cre/Bmal1F/x/- mice (Fig. $3 C$ ), resulting in an overall deletion of BMAL1 across $\sim 70 \%$ of total SCN cells in Drd1a-Cre/Bmal1Flx/mice (Fig. 3B; one-way ANOVA, $F_{(4,22)}=98.83, p<0.0001$, Tukey's post-hoc $p<0.0001$ (vs. Bmal1WT/- or VPAC2-Cre/Bmal1F(x/-))). Notwithstanding this broader deletion of BMAL1, the rest/activity rhythms of these mice were comparable to those of the control 
groups: only 3 of 7 showed minor instability in the time of onset of wheelrunning, but none of them showed a phenotype comparable to those of VPAC2Cre/Bmal1Flx/- mice (Fig. 4G), and the relative amplitudes of their rhythms were not significantly different from control groups (Fig. 4E; Dunn's post hoc $p>$ 0.9999 (vs. Bmal1Flx/-)). These results emphasize that the effects of deletion of BMAL1 from VPAC2 cells are not merely due to reaching a threshold number of SCN neurons, given that the Drd1a-Cre-mediated removal targeted over twice as many cells as that of VPAC2-Cre. Rather, they suggest that the nature and identity of the cells from which BMAL1 is deleted (in this case VPAC2 or Drd1a) are paramount.

\section{The cell-autonomous clock of VPAC2 cells is essential for molecular pacemaking in the SCN.}

The loss of behavioural rhythms in conditionally BMAL1-deleted mice could indicate that a functional cell-autonomous TTFL in VPAC2 cells is required for either producing a coherent ensemble signal within the SCN circuit or for enabling distribution of an appropriate output signal from the SCN to relevant brain centres. To test this, SCN slices were prepared from adult mice following the recording of wheel-running rhythms to establish if behavioural phenotypes were reflected by, and thus likely resulted from, VPAC2-mediated changes in SCN rhythmicity. Circadian rhythms of PER2-Luc bioluminescence from slices lacking either the floxed Bmal1 allele or VPAC2-Cre were stable and of high amplitude (Fig. 5A,B). In contrast, VPAC2-Cre/Bmal1Flx/- SCN slices were highly disorganised, showing erratic and unstable bioluminescence (Fig. 5A), 
A
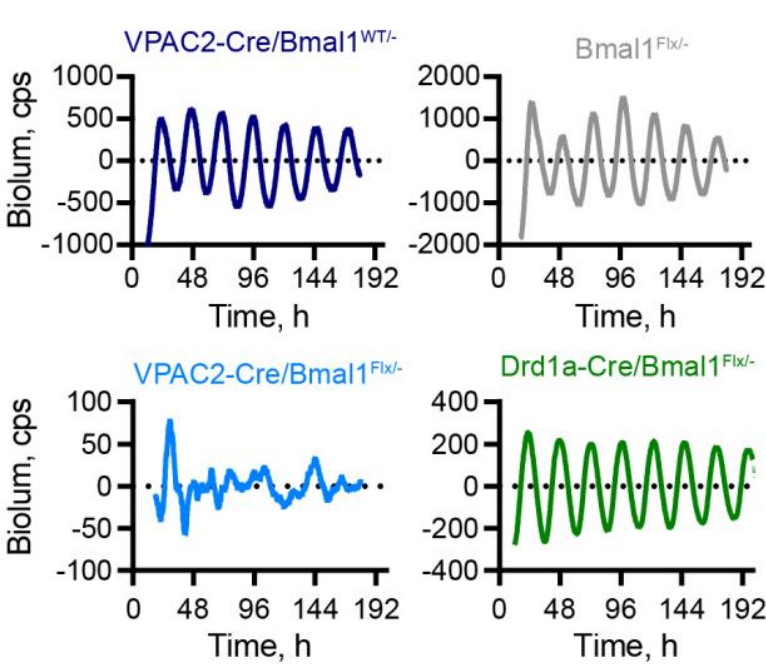

Drd1a-Cre/Bmal1F|x|-

B
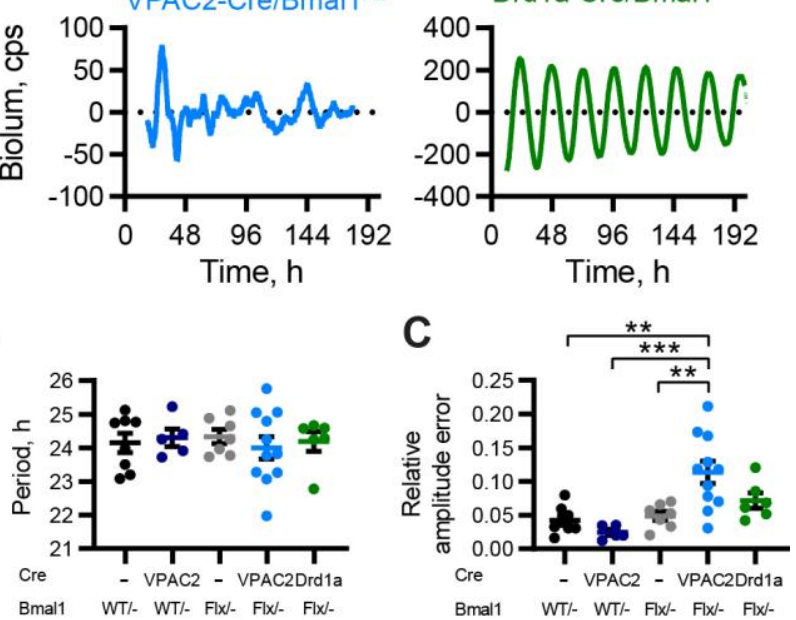

Bmal1 WT/- WT/ Flx/- Flx/- Flx/-

D
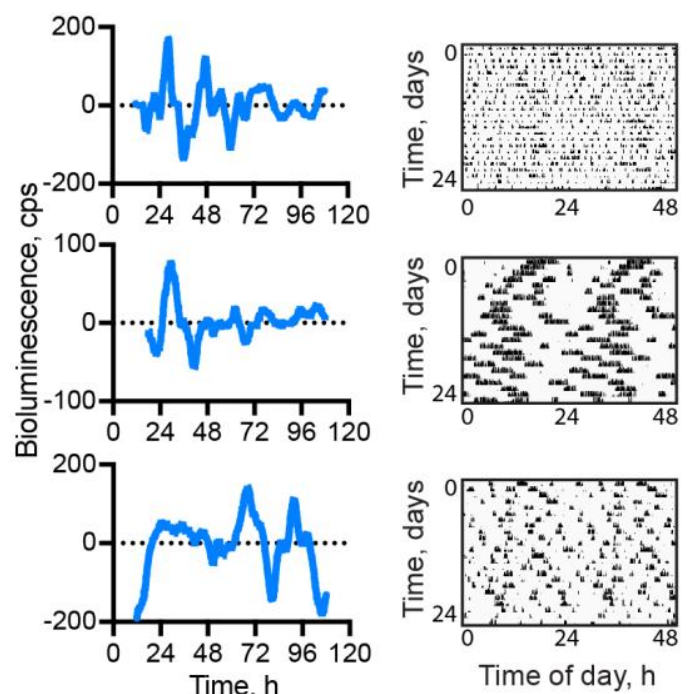

Figure 5. Deletion of BMAL1 from VPAC2-Cre-expressing cells compromises SCN molecular pacemaking.

$\boldsymbol{A}$, Representative, baseline corrected PER2::LUCIFERASE bioluminescence traces from control, VPAC2-Cre/Bmal/ $1^{f \mid x /}$, and Drd1a-Cre/Bmal/f/x/SCN dissected in dim red light following recording of wheel-running rhythms in DD. $B$, Period (mean \pm SEM) of the first 4 bioluminescent

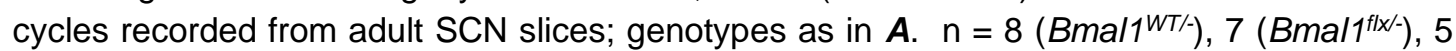

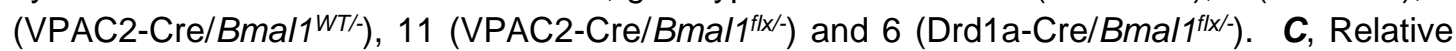
Amplitude Error (RAE) scores (mean \pm SEM) for bioluminescent recordings from SCN slices. $n$ as in $\boldsymbol{B}$. $\boldsymbol{D}$, Representative PER2::LUCIFERASE bioluminescence rhythms from VPAC2$\mathrm{Cre} / \mathrm{Bmal} \mathbf{1}^{f \mid x /}-\mathrm{SCN}$ slices alongside respective actograms from corresponding mice. All tests one-way ANOVA, with Tukey's test for multiple comparisons. ${ }^{*} p<0.05,{ }^{* *} p<0.01,{ }^{* * *} p<$ 0.001 . 
and having significantly higher relative amplitude error (RAE) scores compared to controls (Fig. 5C; one-way ANOVA, $F(4,32)=8.147, p=0.0001$, Tukey's posthoc $p=0.005$ (vs. Bmal1Fl//) $), p=0.0004$ (vs. VPAC2Cre/Bmal1wT//)). The circadian behaviour of VPAC2-Cre/Bmal1F/x/- mice was therefore directly reflected in the lack of competence of molecular pacemaking of the TTFL SCN rhythm: a disrupted, unstable behavioural rhythm was predictive of a poorly organised rhythm in the SCN from the same animal (Fig. 5D). In contrast to the disruption following deletion of BMAL1 from VPAC2 cells, but consistent with minor behavioural effects, the more extensive Drd1a-Cre-mediated deletion of BMAL1 had little impact on the SCN rhythms. They were well defined (Fig. 5A) with a period (Fig. 5B; one-way ANOVA, $F_{(4,32)}=1.425, p=$ 0.93) and a low RAE score comparable to those of the control groups (Fig. 5 C; Tukey's post-hoc $p=0.77$ (vs. Bmal1Flx/-), $p=0.54$ (vs. Bmal1WT/-)). The competence of their SCN was therefore consistent with their well organised wheel-running behaviour. These results confirm the specificity of the contribution of VPAC2-expressing cells to the ensemble rhythm and circadian behaviour, whereby the ablation of the cell-autonomous clock of VPAC2 cells is sufficient to abrogate $\mathrm{SCN}$, and thence behavioural, rhythmicity. This highlights VPAC2-expressing cells as necessary determinants of SCN circadian output and behavioural regulation. 


\section{Discussion}

The potency of the SCN as circadian pacemaker arises from network-level interactions that confer on it the emergent properties essential to its role. The SCN is, however, a heterogeneous mix of neuropeptidergic cell-types and so identifying the particular contribution(s) of defined cell populations to circuitlevel function(s) is an important problem in circadian neurobiology. Here, we used intersectional genetics to manipulate the cell-autonomous clockwork of cells that express the VPAC2 receptor for VIP, and thereby demonstrated that these cells control circadian behaviour and molecular pacemaking within the SCN. These cells therefore constitute an important element of the network topology of the SCN, acting downstream of retinal and VIP-mediated signalling to maintain local and distant circadian coherence.

The ability of VPAC2 cells to determine behavioural circadian period complements results from other intersectional studies. Whereas lengthening the period of VIP cells (Vip-Clock ${ }^{\Delta 19}$ ) had no effect on free-running rhythmicity, the same manipulation in NMS cells (NMS-Clock ${ }^{\Delta 19}$ ), which include almost all VIP and AVP cells, lengthened behavioural period (Lee et al., 2015). Similarly, period-lengthening in Drd1a-Cre cells (including $~ 60 \%$ of AVP cells) in Drd1aCre/Ck1 $1 \varepsilon^{\text {Tau/Tau }}$ mice concomitantly lengthened their behavioural period (Smyllie et al., 2016). Finally, directly altering the period of AVP cells alone accordingly shortened or lengthened behavioural rhythms (Mieda et al., 2016). Given that VPAC2 cells encompass $~ 85 \%$ of AVP cells, the current period effects likely arise from altering cell-autonomous oscillators of VPAC2/AVP 
cells. Moreover, comparable effects can be seen from targetting AVP or VPAC2 cells in ex vivo preparations, insofar as the SCN period does not recapitulate behavioural periods. For example, in vivo period-lengthening of AVP cells to $26 \mathrm{~h}$ disappeared after the first cycle ex vivo, when SCN slices reverted to a wild-type $24 \mathrm{~h}$. Nevertheless, dissection of core and shell SCN allowed the shell AVP cells to express their long cell-autonomous period, confirming effective genetic manipulation (Mieda et al., 2016). Ex vivo, SCN AVP cells are therefore less potent in period-setting than in vivo, similar to VPAC2-targetted SCN. Interestingly, however, VPAC2-Cre/Ck1 $1 \varepsilon^{\text {Tau/- }}$ slices showed a progressive lengthening of period, not seen in control slices, which happened without loss of precision or amplitude. This suggests that, with extended culture, the longer-period VPAC2 cells gained some influence over ensemble period, indicative of circuit-level plasticity.

Overall, therefore, the ability of VPAC2/AVP cells to determine circadian period is greatly enhanced in the intact animal compared to slices. While slice period variability may arise from technical factors such as Cre excision efficiency, the marked, consistent behavioural effect confirmed effective deletion. It is possible that VPAC2 cells function primarily as output cells of the SCN rather than dictating SCN periodicity per se, so their cell-intrinsic period is reflected at the behavioural level but not in slices. This would, however, require two separately oscillating populations within the SCN, which would be expected to lead to more unstable behavioural patterns than were observed. Instead, it is likely that modification of SCN function by behavioural state feed-back, at the level of the entire circuit or directly to the AVP and/or VPAC2 cells, underlies 
these differences. A precedent for behavioural feedback potentiating a compromised SCN is seen in arrhythmic VPAC2-null mice, which, after entrainment to a $24 \mathrm{~h}$ schedule of voluntary exercise, exhibit strong, freerunning circadian behaviour on release into DD (Power et al., 2010). In the case of VPAC2-Cre/Ck1 $1 \varepsilon^{\text {Tau/- }}$ mice, it is likely that the sparse VPAC2 cells in the rest of the hypothalamus that receive input from the SCN, such as in the paraventricular nucleus (Kalló et al., 2004), would also have 24 h periods and may feed-back to, and resonate with, the intrinsically $24 \mathrm{~h}$ SCN VPAC2 cells. This feedback would reinforce the role of SCN VPAC2 cells in perioddetermination in vivo, leading to a change in behaviour, but would be absent in the slice.

In addition to their period-setting abilities, VPAC2 cells are also critical for rhythm maintenance, as evidenced by BMAL1 deletion in this cell population. Interestingly, the total number of targetted cells appears to be less important than the type of cell rendered circadian incompetent. In pan-neuronal deletion studies, loss of BMAL1 from $\sim 30 \%$ of neurons using a Nestin-Cre line did not have any effect on behavioural rhythms in DD (Mieda and Sakurai, 2011). Equally, deletion of BMAL1 in $65 \%$ of SCN cells using a single allele of Synaptotagmin10-Cre had no behavioural effect (Husse et al., 2011). Only in homozygous Synaptotagmin10-Cre mice, where BMAL1 was deleted from $83 \%$ of the SCN, was a behavioural phenotype evident. Moreover, in the current study loss of BMAL1 from Drd1a-expressing cells, which caused $\sim 70 \%$ loss of BMAL1 across the SCN, did not compromise circadian behaviour. Conversely, deletion of BMAL1 from NMS neurons, which constitute a minority of $40 \%$ of 
the SCN, nevertheless caused behavioural arrhythmia. In the current study, loss of BMAL1 from only $15-20 \%$ of total SCN cells, but $70 \%$ of VPAC2 cells, severely disrupted circadian behaviour. Unlike in pacemaking, where VPAC2 and AVP share many commonalities, loss of BMAL1 exclusively from AVP cells did not cause disrupted circadian behaviour, instead causing the nocturnal activity profile to widen and overall period to lengthen by $\sim 1 \mathrm{~h}$ (Mieda et al., 2015). Thus, VPAC2 cells appear to constitute a population in which rhythmicity is essential for overall circadian competence.

Further demonstration of the essential role of VPAC2 cells came from the ex vivo SCN slices, which exhibited highly disordered circadian cycles of TTFL activity, commensurate with the loss of behavioural coherence in these mice. This contrasts with the sustained TTFL function following the ablation of BMAL1 solely from AVP cells (Mieda et al., 2015), and from Drd1a-Cre cells in the current study. This suggests that the loss of behavioural coherence seen in VPAC2-Cre/Bmal/flx/- mice has a more fundamental origin than simply an inability to convey behaviourally relevant circadian cues outside the SCN (a deficiency seen with loss of Prok2 receptor (Prosser et al., 2007). Even more strikingly, Ko et al. (2010) demonstrated that in global Bmal1/- slices, stochastic quasi-circadian rhythmicity can be seen, thus it is remarkable that loss of BMAL1 from $20 \%$ of the SCN can cause such a pronounced phenotype. This provides strong evidence that VPAC2 cells in the SCN exert powerful control over ensemble rhythmicity, a result complementing those of Patton et al. (2020), together showing that a competent TTFL in VPAC2 cells is necessary for coherent circadian oscillation. 
Notwithstanding the overall effect of BMAL1 deletion, it is clear that the behavioural phenotype of VPAC2-Cre/Bmal/1/x/- mice was variable both in severity and the time that it took to be expressed under DD, suggesting that there are sub-populations of VPAC2 cells, as posited for other SCN populations (Kawamoto et al., 2003; Geoghegan and Carter, 2008). The advent of single cell transcriptomic profiling (Park et al., 2016; Wen et al., 2020) may reveal suitable markers for such sub-populations. That the behavioural disruption took time to emerge is intriguing, because, as with NMS-BMAL1 mice, phenotypes often appeared suddenly, with no obvious trigger, suggesting "the presence of mechanisms capable of transiently compensating for the loss of molecular clocks" (Lee et al., 2015). The initial rhythmicity in vivo, perhaps initiated by daily lighting cycles and/or sustained by light-driven behavioural rhythms, may have relied on coupling effects of non-VIP-ergic cells that compensated for the loss of cell-autonomous oscillators in the VPAC2 cells. Potential compensatory factors are AVP and GRP, both of which have been shown to be capable of inducing rhythmicity in VIP- or VPAC2-deficient SCN (Brown et al., 2005; Maywood et al., 2006, 2011). Levels of AVP, however, were reduced in VPAC2-Cre/Bmal $1^{f \mid x /-}$ mice, which may have exacerbated circadian disorganisation arising from loss of TTFL function in VPAC2 cells. The signalling molecules used by VPAC2 cells themselves remain unclear, not least because a global AVP knockout has only a minor effect on rhythmicity (Groblewski et al., 1981), although loss of AVP receptors does compromise intercellular coupling in the SCN (Yamaguchi et al., 2013). Similarly, NMS, 
another peptide marker for both core and shell SCN, is not essential for circadian function (Lee et al., 2015).

In summary, we have demonstrated that VPAC2 cells constitute an essential pace-setting and rhythm-generating population of the mammalian circadian system, necessary for fully coherent circadian oscillation both in vivo and ex vivo. Furthermore, we found that the strong pace-setting abilities of VPAC2 cells are disrupted, albeit not completely absent, on slice preparation, suggesting an unanticipated influence of extra-SCN populations in reinforcing periodicity. These findings complement those of Patton et al. (2020) that circadian competence in both VPAC2 cells and VIP cells is necessary for the de novo initiation of ensemble rhythms in SCN slices and behaviour. Thus, circadian competence in VPAC2 cells is necessary for rhythmicity, but they require their cognate signalling cell partners to be sufficient for rhythmicity. The VIPergic axis confers much of the fundamental robustness and intercellular communication that is essential to normal SCN function, and our work advances our knowledge of the individual functions of the cells inherent to this axis. 


\section{References}

Abrahamson EE, Moore RY (2001) Suprachiasmatic nucleus in the mouse: retinal innervation, intrinsic organization and efferent projections. Brain Res 916:172-191.

Antle MC, Silver R (2005) Orchestrating time: Arrangements of the brain circadian clock. Trends Neurosci 28:145-151.

Aton SJ, Colwell CS, Harmar AJ, Waschek J, Herzog ED (2005) Vasoactive intestinal polypeptide mediates circadian rhythmicity and synchrony in mammalian clock neurons. Nat Neurosci 8:476-483.

Brancaccio M, Patton AP, Chesham JE, Maywood ES, Hastings MH (2017) Astrocytes Control Circadian Timekeeping in the Suprachiasmatic Nucleus via Glutamatergic Signaling. Neuron 93:1-16.

Brown TM, Hughes AT, Piggins HD (2005) Gastrin-releasing peptide promotes suprachiasmatic nuclei cellular rhythmicity in the absence of vasoactive intestinal polypeptide-VPAC2 receptor signaling. J Neurosci 25:11155-11164.

Bunger MK, Wilsbacher LD, Moran SM, Clendenin C, Radcliffe LA, Hogenesch JB, Simon MC, Takahashi JS, Bradfield CA (2000) Mop3 is an essential component of the master circadian pacemaker in mammals. Cell 103:1009-1017.

Ciarleglio CM, Gamble KL, Axley JC, Strauss BR, Cohen JY, Colwell CS, McMahon DG (2009) Population encoding by circadian clock neurons organizes circadian behavior. J Neurosci 29:1670-1676. 
Colwell CS, Michel S, Itri J, Rodriguez W, Tam J, Lelievre V, Hu Z, Liu X, Waschek JA (2003) Disrupted circadian rhythms in VIP- and PHIdeficient mice. Am J Physiol Regul Integr Comp Physiol 285:R939-49.

Geoghegan D, Carter DA (2008) A novel site of adult doublecortin expression: neuropeptide neurons within the suprachiasmatic nucleus circadian clock. BMC Neurosci 9:1-9.

Gibbs JE, Blaikley J, Beesley S, Matthews L, Simpson KD, Boyce SH, Farrow SN, Else KJ, Singh D, Ray DW, Loudon ASI (2012) The nuclear receptor REV-ERBa mediates circadian regulation of innate immunity through selective regulation of inflammatory cytokines. Proc Natl Acad Sci 109:582-587.

Groblewski TA, Nunez AA, Gold RM (1981) Circadian rhythms in vasopressin deficient rats. Brain Res Bull 6:125-130.

Hamnett R, Crosby P, Chesham JE, Hastings MH (2019) Vasoactive intestinal peptide controls the suprachiasmatic circadian clock network via ERK1/2 and DUSP4 signalling. Nat Commun 10:542.

Harmar AJ, Marston HM, Shen S, Spratt C, West KM, Sheward WJ, Morrison CF, Dorin JR, Piggins HD, Reubi JC, Kelly JS, Maywood ES, Hastings MH (2002) The VPAC2 receptor is essential for circadian function in the mouse suprachiasmatic nuclei. Cell 109:497-508.

Hastings MH, Maywood ES, Brancaccio M (2018) Generation of circadian rhythms in the suprachiasmatic nucleus. Nat Rev Neurosci 19:453-469.

Hastings MH, Reddy AB, McMahon DG, Maywood ES (2005) Analysis of 
circadian mechanisms in the suprachiasmatic nucleus by transgenesis and biolistic transfection. Methods Enzymol 393:579-592.

Husse J, Zhou X, Shostak A, Oster H, Eichele G (2011) Synaptotagmin10Cre, a driver to disrupt clock genes in the SCN. J Biol Rhythms 26:379389.

Jin X, Shearman LP, Weaver DR, Zylka MJ, De Vries GJ, Reppert SM (1999) A molecular mechanism regulating rhythmic output from the suprachiasmatic circadian clock. Cell 96:57-68.

Jones JR, Simon T, Lones L, Herzog ED (2018) SCN VIP neurons are essential for normal light-mediated resetting of the circadian system. J Neurosci 38:7986-7995.

Jones JR, Tackenberg MC, McMahon DG (2015) Manipulating circadian clock neuron firing rate resets molecular circadian rhythms and behavior. Nat Neurosci 18:373-375.

Kalló I, Kalamatianos T, Wiltshire N, Shen S, Sheward W, Harmar AJ, Coen CW (2004) Transgenic approach reveals expression of the VPAC2 receptor in phenotypically defined neurons in the mouse suprachiasmatic nucleus and in its efferent target sites. Eur J Neurosci 19:2201-2211.

Kawamoto K, Nagano M, Kanda F, Chihara K, Shigeyoshi Y, Okamura H (2003) Two types of VIP neuronal components in rat suprachiasmatic nucleus. J Neurosci Res 74:852-857.

Ko CH, Yamada YR, Welsh DK, Buhr ED, Liu AC, Zhang EE, Ralph MR, Kay SA, Forger DB, Takahashi JS (2010) Emergence of noise-induced 
oscillations in the central circadian pacemaker. PLoS Biol 8:e1000513.

Krempels K, Usdin TB, Harta G, Mezey E (1995) PACAP acts through VIP type 2 receptors in the rat testis. Neuropeptides 29:315-320.

Lee IT, Chang AS, Manandhar M, Shan Y, Fan J, Izumo M, Ikeda Y, Motoike T, Dixon S, Seinfeld JE, Takahashi JS, Yanagisawa M (2015)

Neuromedin S-Producing Neurons Act as Essential Pacemakers in the Suprachiasmatic Nucleus to Couple Clock Neurons and Dictate Circadian Rhythms. Neuron 85:1086-1102.

Liu AC, Welsh DK, Ko CH, Tran HG, Zhang EE, Priest AA, Buhr ED, Singer O, Meeker K, Verma IM, Doyle FJ, Takahashi JS, Kay SA (2007) Intercellular coupling confers robustness against mutations in the SCN circadian clock network. Cell 129:605-616.

Luo L et al. (2020) Optimizing Nervous System-Specific Gene Targeting with Cre Driver Lines: Prevalence of Germline Recombination and Influencing Factors. Neuron 106:1-29.

Maywood E, Chesham JE, O’Brien JA, Hastings MH (2011) A diversity of paracrine signals sustains molecular circadian cycling in suprachiasmatic nucleus circuits. Proc Natl Acad Sci U S A 108:14306-14311.

Maywood ES, Reddy AB, Wong GKY, O’Neill JS, O’Brien JA, McMahon DG, Harmar AJ, Okamura H, Hastings MH (2006) Synchronization and maintenance of timekeeping in suprachiasmatic circadian clock cells by neuropeptidergic signaling. Curr Biol 16:599-605.

Mazuski C, Abel JH, Chen SP, Hermanstyne TO, Jones JR, Simon T, Doyle 
FJ, Herzog ED (2018) Entrainment of Circadian Rhythms Depends on

Firing Rates and Neuropeptide Release of VIP SCN Neurons. Neuron 99:555-563.

McDearmon EL, Patel KN, Ko CH, Walisser JA, Schook AC, Chong JL, Wilsbacher LD, Song EJ, Hong H-K, Bradfield CA, Takahashi JS (2006) Dissecting the functions of the mammalian clock protein BMAL1 by tissue-specific rescue in mice. Science 314:1304-1308.

Meng QJ, Logunova L, Maywood ES, Gallego M, Lebiecki J, Brown TM, Sládek M, Semikhodskii AS, Glossop NRJ, Piggins HD, Chesham JE, Bechtold DA, Yoo SH, Takahashi JS, Virshup DM, Boot-Handford RP, Hastings MH, Loudon ASI (2008) Setting Clock Speed in Mammals: The CK1ع tau Mutation in Mice Accelerates Circadian Pacemakers by Selectively Destabilizing PERIOD Proteins. Neuron 58:78-88.

Mieda M, Okamoto H, Sakurai T (2016) Manipulating the Cellular Circadian Period of Arginine Vasopressin Neurons Alters the Behavioral Report Manipulating the Cellular Circadian Period of Arginine Vasopressin Neurons Alters the Behavioral Circadian Period. Curr Biol 26:1-8.

Mieda M, Ono D, Hasegawa E, Okamoto H, Honma K, Honma S, Sakurai T (2015) Cellular Clocks in AVP Neurons of the SCN Are Critical for Interneuronal Coupling Regulating Circadian Behavior Rhythm. Neuron 85:1103-1116.

Mieda M, Sakurai T (2011) Bmal1 in the Nervous System Is Essential for Normal Adaptation of Circadian Locomotor Activity and Food Intake to 
Periodic Feeding. J Neurosci 31:15391-15396.

Park J, Zhu H, O'Sullivan S, Ogunnaike BA, Weaver DR, Schwaber JS, Vadigepalli R (2016) Single-cell transcriptional analysis reveals novel neuronal phenotypes and interaction networks involved in the central circadian clock. Front Neurosci 10:1-19.

Patton AP, Chesham JE, Hastings MH (2016) Combined Pharmacological and Genetic Manipulations Unlock Unprecedented Temporal Elasticity and Reveal Phase-Specific Modulation of the Molecular Circadian Clock of the Mouse Suprachiasmatic Nucleus. J Neurosci 36:9326-9341.

Patton AP, Edwards MD, Smyllie NJ, Hamnett R, Chesham JE, Brancaccio M, Maywood ES, Hastings MH (2020) The VIP-VPAC2 neuropeptidergic axis is a cellular pacemaking hub of the suprachiasmatic nucleus circadian circuit. Nat Commun 11:3394.

Power A, Hughes ATL, Samuels RE, Piggins HD (2010) Rhythm-Promoting Actions of Exercise in Mice with Deficient Neuropeptide Signaling. J Biol Rhythms 25:235-246.

Prosser HM, Bradley A, Chesham JE, Ebling FJP, Hastings MH, Maywood ES (2007) Prokineticin receptor 2 (Prokr2) is essential for the regulation of circadian behavior by the suprachiasmatic nuclei. Proc Natl Acad Sci U S A 104:648-653.

Reddy AB, Karp NA, Maywood ES, Sage EA, Deery M, O’Neill JS, Wong GKY, Chesham J, Odell M, Lilley KS, Kyriacou CP, Hastings MH (2006) Circadian Orchestration of the Hepatic Proteome. Curr Biol 16:1107- 
1115.

Reppert SM, Weaver DR (2002) Coordination of circadian timing in mammals. Nature 418:935-941.

Smyllie NJ, Chesham JE, Hamnett R, Maywood ES, Hastings MH (2016)

Temporally chimeric mice reveal flexibility of circadian period-setting in the suprachiasmatic nucleus. Proc Natl Acad Sci 113:3657-3662.

Srinivas S, Watanabe T, Lin CS, William CM, Tanabe Y, Jessell TM, Costantini F (2001) Cre reporter strains produced by targeted insertion of EYFP and ECFP into the ROSA26 locus. BMC Dev Biol 1.

Usdin T, Bonner T, Mezey E (1994) Two Receptors for Vasoactive Intestinal Polypeptide with Similar Specificity and Complementary Distributions. Endocrinology 135:2662-2680.

Wen S, Ma D, Zhao M, Xie L, Wu Q, Gou L, Zhu C, Fan Y, Wang H, Yan J (2020) Spatiotemporal single-cell analysis of gene expression in the mouse suprachiasmatic nucleus. Nat Neurosci 23:456-467.

Yamaguchi Y, Suzuki T, Mizoro Y, Kori H, Okada K, Chen Y, Fustin J-M, Yamazaki F, Mizuguchi N, Zhang J, Dong X, Tsujimoto G, Okuno Y, Doi M, Okamura H (2013) Mice genetically deficient in vasopressin V1a and V1b receptors are resistant to jet lag. Science 342:85-90.

Yoo S-H, Yamazaki S, Lowrey PL, Shimomura K, Ko CH, Buhr ED, Siepka SM, Hong H-K, Oh WJ, Yoo OJ, Menaker M, Takahashi JS (2004) PERIOD2::LUCIFERASE real-time reporting of circadian dynamics reveals persistent circadian oscillations in mouse peripheral tissues. Proc 
Natl Acad Sci U S A 101:5339-5346.

Zielinski T, Moore AM, Troup E, Halliday KJ, Millar AJ (2014) Strengths and limitations of period estimation methods for circadian data. PLoS One 9:e96462. 